\title{
Effectiveness and safety of the interleukin 6-receptor antagonist tocilizumab after 4 and 24 weeks in patients with active rheumatoid arthritis: the first phase Illb real-life study (TAMARA)
}

\author{
Gerd R Burmester, ${ }^{1}$ E Feist, ${ }^{1}$ H Kellner, ${ }^{2} \mathrm{~J}$ Braun, ${ }^{3} \mathrm{C}$ Iking-Konert, ${ }^{4}$ A Rubbert-Roth ${ }^{5}$
}

- Additional data are published online only. To view these files please visit the journal online at (http://ard.bmj.com)

${ }^{1}$ Charité-Universitätsmedizin Berlin, Department of Rheumatology and Clinical Immunology, Free University and Humboldt University of Berlin,

Berlin, Germany

${ }^{2}$ Rheumatologische Praxis,

München, Germany

${ }^{3}$ Rheumazentrum Ruhrgebiet

Herne, Herne, Germany

${ }^{4}$ Department III, Nephrology and Rheumatology, University

Clinic Hamburg UKE, Hamburg, Germany

${ }^{5}$ Department I, Internal Medicine, University of Cologne, Cologne, Germany

\section{Correspondence to}

Gerd-R Burmester, Charité-

Universitätsmedizin Berlin,

Department of Rheumatology

and Clinical Immunology,

Free University and Humboldt

University of Berlin, Charitéplatz

1, 10117 Berlin;

gerd.burmester@charite.de

Accepted 11 November 2010

Published Online First

27 December 2010

\section{ABSTRACT}

Objectives To confirm the effectiveness and safety of the interleukin 6-receptor antagonist tocilizumab in patients with rheumatoid arthritis (RA) in a setting close to real-life medical care in Germany.

Methods A multicentre open-label phase Illb study was undertaken. Patients with active RA with a 28-joint Disease Activity Score (DAS28) > 3.2 despite previous disease-modifying antirheumatic drugs (DMARDs) were treated with tocilizumab $8 \mathrm{mg} / \mathrm{kg}$ every 4 weeks. The primary end point was the proportion of patients achieving LDAS $\leq 3.2$ at week 24; secondary end points included American College of Rheumatology (ACR), European League Against Rheumatism (EULAR) or Clinical Disease Activity Index (CDAl) responses and decrease in acute phase. Analyses in subgroups such as rheumatoid factor (RF)-positive versus RF-negative patients and patients with an inadequate response to treatment with DMARDs (DMARD-IR) versus those with an inadequate response to tumour necrosis factor (TNF) antagonists (TNF antagonist-IR) were performed. Safety was assessed by adverse event documentation.

Results 286 patients were treated and $83.6 \%$ completed the study. $41.6 \%$ had previously been treated with TNF antagonists. $57 \%$ of the intention-to-treat patients achieved the primary end point of LDAS, $47.6 \%$ achieved DAS remission $<2.6$ and a EULAR 'good response' was achieved by $54.9 \%$; ACR50/70 response rates at week 24 were $50.7 \%$ and $33.9 \%$, respectively. The mean \pm SD decrease in CDAl from baseline to week 24 was $71 \pm 29 \%$. C reactive protein levels normalised rapidly within 1 week. Major improvements in fatigue, pain and morning stiffness were observed in the first 4 weeks and further improved until week 24. DAS28, EULAR and ACR responses at week 24 did not differ between RF-positive and RF-negative patients. TNF antagonist-naive patients responded better than patients who had previously failed on TNF antagonists. The safety profile of tocilizumab was comparable to that previously observed in the phase III trial programme. Serious infections were observed in $3.1 \%$ of patients.

Conclusions Tocilizumab is highly effective in a setting close to real-life medical care with a rapid and sustained improvement in signs and symptoms of RA. A manageable safety profile was seen over the 24-week study period.

\section{INTRODUCTION}

This paper is freely available online under the BMJ Journals unlocked scheme, see http:// ard.bmj.com/info/unlocked.dtl
During the past years the treatment of rheumatoid arthritis (RA) has changed considerably. ${ }^{1}{ }^{2}$ In addition to treatment with conventional diseasemodifying antirheumatic drugs (DMARDs), biological agents have emerged with the capability of specifically targeting single components within the inflammatory cascade ${ }^{3}$ such as inhibiting tumour necrosis factor (TNF) $\alpha^{4}$ or interleukin (IL) $-1,{ }^{5}$ targeting $\mathrm{CD} 20 \mathrm{~B}^{\text {cells }}{ }^{6}$ or interfering with $\mathrm{T}$ cell activation by blocking CD80/86:CD28 signalling. However, approximately $70 \%$ of patients still fail to achieve remission and approximately $29-54 \%$ do not show significant improvement with TNF $\alpha$ antagonists. ${ }^{8-10}$ The development of other innovative targeted therapies with alternative modes of action is therefore needed.

Tocilizumab, a recombinant humanised monoclonal IgG $\mathrm{I}_{1}$ antihuman interleukin 6-receptor antibody represents such a new treatment option in patients with moderate to severe active RA who have either responded inadequately or were intolerant to previous treatment with one or more DMARDs or TNF antagonists.

In clinical studies it was shown that tocilizumab is well tolerated and efficacious in alleviating the signs and symptoms of RA, ${ }^{11-15}$ as well as inhibiting radiological progression. ${ }^{16} 17$ The results of these studies led to its approval by the European Medicines Agency (EMA) in January 2009 and by the FDA in January 2010. The clinical phase II and III studies, however, required strict eligibility criteria and rigid adherence to a comprehensive schedule of studyrelated events. In the phase IIIb study (TAMARA), the efficacy and safety of tocilizumab in a setting closer to daily practice was investigated.

\section{METHODS}

\section{Study design and patients}

TAMARA (Tocilizumab And DMARDs: Achievements in Rheumatoid Arthritis), a multicentre open-label non-controlled single-arm study, was performed at 70 centres in Germany from September 2008 to July 2009. Men and women aged $>18$ years with moderate to severe active RA of $\geq 6$ months' duration who had an inadequate clinical response (28-joint Disease Activity Score (DAS28) >3.2) to a stable dose of conventional or biological DMARDs were included. Patients were treated with tocilizumab $8 \mathrm{mg} / \mathrm{kg}$ every 4 weeks at day 1 and weeks 4, 8, 12, 16 and 20 in addition to their stable background DMARD. The primary outcome was the proportion of patients reaching a DAS $\leq 3.2$ after 24 weeks. Secondary outcomes were improvements in the European 
League Against Rheumatism (EULAR) response, DAS remission, American College of Rheumatology (ACR) responses and the safety of tocilizumab with regard to adverse events (AEs), laboratory assessments and physical examination. In addition, effects on health-related quality of life outcomes in the study population were assessed. Details of the study population, statistics and methods are shown in the online supplement.

\section{RESULTS}

\section{Patients}

After screening of 334 patients, 286 patients were enrolled in the study and $85.6 \%$ completed the 24 -week period and were included in the primary analysis intention-to-treat(ITT) population consisting of $24.5 \%$ men and $75.5 \%$ women aged $18-84$ years All but one patient was pretreated with DMARDs, mostly with methotrexate $(95.1 \%)$ or leflunomide $(60.1 \%)$, and $41.6 \%$ of patients were pretreated with TNF $\alpha$ antagonists. During the study, concomitant methotrexate was used by $72.0 \%$, leflunomide by $19.6 \%$, glucocorticoids by $70.6 \%$ and nine patients received tocilizumab monotherapy (see figure 3 in the online supplement). Deviations from the protocol leading to exclusion from the per protocol population (PP) occurred in 153 patients (deviations with a frequency of $>5 \%$ are listed in figure in the online supplement), but only 11 patients $(3.3 \%)$ had to be withdrawn because of protocol violations. Thus, 133 patients were valid for inclusion in the strict PP population. Overall, the protocol deviations had no
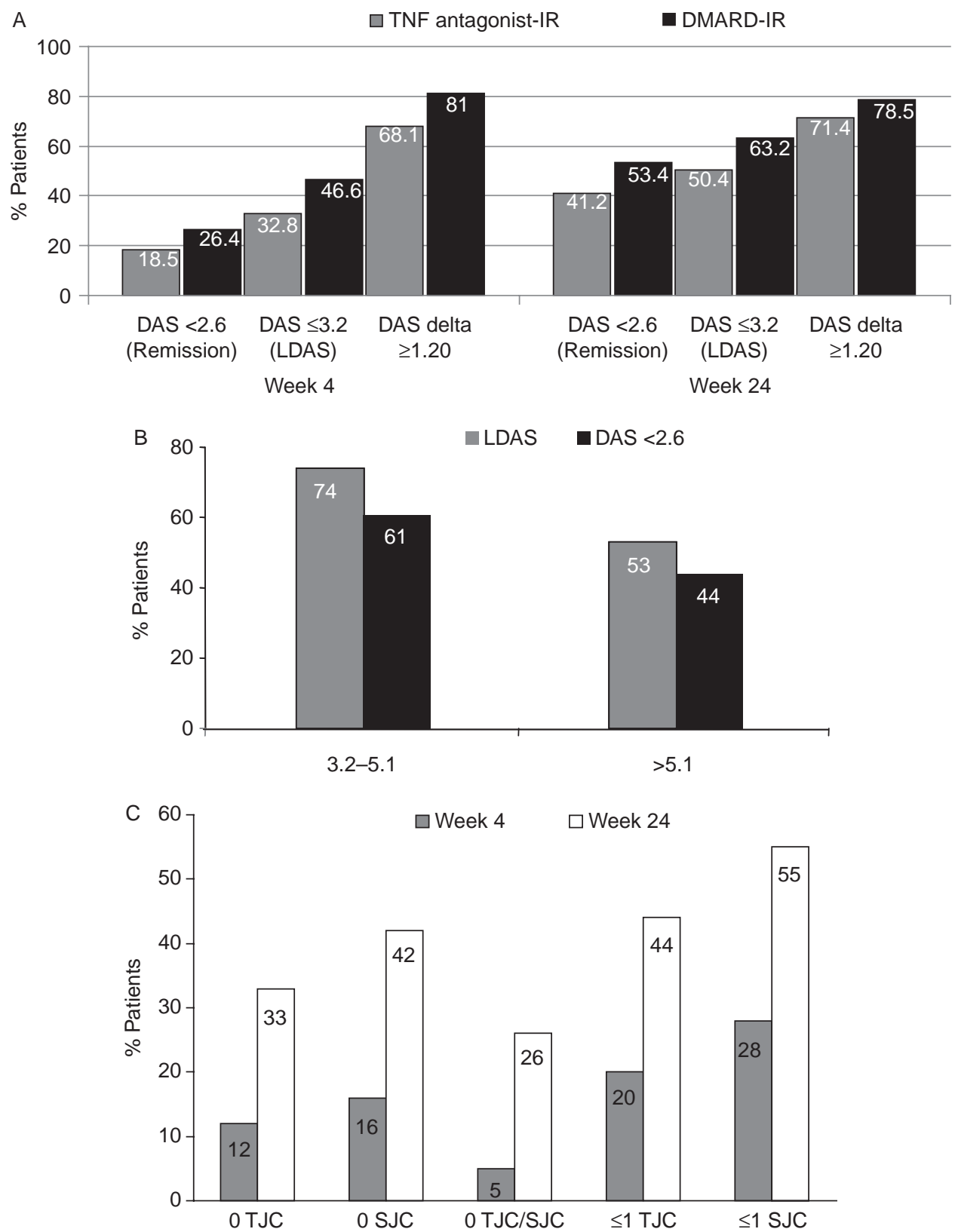

Figure 1 (A) Response categories of DAS28 (DAS remission, LDAS, clinically significant reduction in DAS delta $>1.2$ ) in TNF antagonist-IR and DMARD-IR patients. 'Data as observed', missing data were evaluated as non-response. (B) Change in DAS28 from baseline to week 4 and week 24 (percentage of patients in LDAS and DAS remission after 24 weeks depending on their DAS at baseline). (C) Percentage of patients with no swollen joints and/or no tender joints at weeks 4 and 24 (LOCF). DAS28, 28-joint Disease Activity Score; DMARD-IR, inadequate response to diseasemodifying antirheumatic drugs; LDAS, Low Disease Activity Score; LCOF, last observation carried forward; SJC, swollen joint count; TJC, tender joint count; TNF antagonist-IR, inadequate response to tumour necrosis factor antagonists. 
detectable influence on the study outcomes; the results of the ITT and PP populations were nearly identical.

\section{Efficacy}

Analysis of the primary efficacy variable showed that 163 of the 286 patients $(57.0 \%)$ in the ITT population achieved a Low Disease Activity Score (LDAS) at week 24 (63.2\% with DMARD pretreatment and $50.4 \%$ with TNF antagonist pretreatment). More DMARD-IR patients (53.4\%) than TNF antagonist-IR patients $(41.2 \%)$ reached DAS remission (figure $1 \mathrm{~A}$ ).

Tocilizumab showed a very early onset of action. The mean DAS28 at baseline was $6.0 \pm 1.0$ points. After the first infusion the mean DAS28 decreased by $21.5 \pm 18.0 \%$ within 1 week and by $40.1 \pm 19.9 \%$ within 4 weeks. $75.2 \%$ of patients experienced a clinically significant reduction by $\geq 1.2$ DAS 28 points, $40.9 \%$ achieved an LDAS and $23.1 \%$ were in DAS28 remission after only one infusion. Thereafter, mean disease activity continuously decreased: by week 24 mean DAS28 decreased from $6.0 \pm 1.0$ points at baseline to $2.6 \pm 1.5$ points (reduction of $56.8 \pm 22.5 \%$ ).

Most of the patients had a high disease activity at baseline; $79.2 \%$ had a DAS28 $>5.1$ which decreased by $16.1 \%$ at week 4 , and only $6.6 \%$ persistently had a DAS $>5.1$ at week 24 while most $(53.0 \%)$ of the patients achieved LDAS and $44 \%$ were in DAS28 remission. Of the patients with a baseline DAS28 between $>3.2$ and $\leq 5.1(n=54), 74 \%$ achieved LDAS and $61 \%$ achieved DAS28 remission at week 24 (figure 1B).

ACR response rates at week 24 showed that $65.0 \%$ of the patients met the criteria for an ACR20 response, $50.7 \%$ for an ACR50 response and $33.9 \%$ for an ACR70 response. These improvements were seen in all components of the ACR core set, notably in the number of swollen and tender joints which significantly decreased in both DMARD-IR and TNF antagonist-IR patients (table 1). After 24 weeks, $33 \%$ of patients had a tender joint count (TJC) of 0 and $42 \%$ had a swollen joint count (SJC) of $0 ; 26 \%$ of patients had both TJC and SJC of 0 (figure 1C). Accordingly, the Clinical Disease Activity Index decreased by $70.9 \pm 29.3 \%$ from $34.7 \pm 12.5$ to $11.3 \pm 11.5$ within 24 weeks.

A subgroup analysis of RF-positive versus RF-negative patients did not show a statistically significant difference in DAS28, EULAR or ACR responses at week 24 (table 2), although RF-positive patients had a higher DAS28 than RF-negative patients at baseline (6.1 vs 5.7). Moreover, age, sex, body mass index, concomitant diseases and pretreatment did not differ between the two groups (data not shown). In addition, the subgroup of patients with concomitant leflunomide therapy achieved a significant clinical improvement $55.4 \%$ EULAR good response, DAS remission in $48.2 \%$, LDAS in $57.1 \%$ ) which was comparable to the results observed in the entire cohort.

The significant results obtained with the DAS28 assessments were confirmed by improvements in all other secondary efficacy parameters. As shown in figure 2A, the self-reported 'patient take home form' indicated that the strongest effects had already appeared within the first 4 weeks of treatment (ie, following the very first infusion) in pain, morning stiffness and fatigue. Also, in other patientreported outcomes, improvement in health-related quality of life measured by the Health Assessment Questionnaire-Disease Index (HAQ-DI) was reported (figure 2B), and also in the Short Form-36 (SF-36) which showed an improvement in physical functioning and vitality as well as in social functioning and mental health.

The mean global satisfaction of patients with the treatment according to the Treatment Satisfaction Questionnaire for Medication was $74.7 \pm 25.9 \%$. At week $24,72.8 \%$ of patients in the DMARD-IR subgroup and $65.5 \%$ of patients in the TNF antagonist-IR subgroup were satisfied with the effectiveness of the treatment and $89.7 \%$ in the DMARD-IR subgroup and $88.5 \%$ in the TNF antagonist-IR subgroup were satisfied with the tolerability. Global satisfaction with the medication was reported by $77.8 \%$ of patients in the DMARD-IR subgroup and $71.6 \%$ of patients in the TNF antagonist-IR subgroup.

\section{Safety}

The safety profile of tocilizumab was comparable to that previously observed. A total of 242 patients (84.6\%) experienced at least one AE during the study. Most of the AEs were mild or moderate and resolved by the end of the study. No deaths were reported. Sixteen patients $(5.6 \%)$ were withdrawn from treatment due to AEs and 10 (3.5\%) due to study drug-related AEs. Table 3 in the online supplement shows the most common study drug-related AEs and differences in the incidence of AEs between patients pretreated with DMARDs or TNF antagonists.

Fifteen patients (5.2\%) had serious AEs related to the study drug, which were mostly infections. In nine patients with infections $(3.1 \%)$ at least one infection was serious (see table 3 in online supplement for details). Three patients prematurely discontinued the study due to serious infections. No serious AEs related to the study drug occurred at frequencies $\geq 1 \%$.

AEs of special interest occurred in 156 patients $(54.5 \%)$ and in $120(42.0 \%)$ at least one was rated as drug-related. The highest incidence rates were seen in lipid elevations $(29.7 \%$ in total; $23.4 \%$ drug-related) and infections $(28.7 \%$ in total; $17.8 \%$ drugrelated). The mean changes in the lipid panel (total cholesterol, triglycerides, high-density lipoprotein and low-density lipoprotein) indicated mild increases and only in a few patients $(n=15)$

Table 1 American College of Rheumatology core set components at baseline, week 4 and week 24 (last observation carried forward) in TNF antagonist-IR and DMARD-IR subgroups of patients

\begin{tabular}{|c|c|c|c|c|c|c|}
\hline \multirow[b]{2}{*}{ Component } & \multicolumn{2}{|l|}{ Baseline } & \multicolumn{2}{|l|}{ Week 4} & \multicolumn{2}{|l|}{ Week 24} \\
\hline & TNF antagonist-IR & DMARD-IR & TNF antagonist-IR & DMARD-IR & TNF antagonist-IR & DMARD-IR \\
\hline$\overline{S J C / 28 ~(n) ~}$ & $12.3 \pm 8.4$ & $13.3 \pm 7.3$ & $6.6 \pm 5.9$ & $6.2 \pm 7.1$ & $3.8 \pm 4.9$ & $3.0 \pm 4.9$ \\
\hline TJC/28 (n) & $20.7 \pm 14.2$ & $20.0 \pm 11.2$ & $13.1 \pm 13.1$ & $10.3 \pm 11.4$ & $8.7 \pm 11.7$ & $5.3 \pm 8.4$ \\
\hline $\mathrm{ESR}(\mathrm{mm} / \mathrm{h})$ & $40.5 \pm 24.4$ & $35.6 \pm 20.3$ & $11.7 \pm 14.8$ & $9.3 \pm 12.5$ & $7.2 \pm 10.1$ & $6.9 \pm 9.1$ \\
\hline VAS physician disease activity (mm) & $64.6 \pm 16.6$ & $61.2 \pm 19.4$ & $38.1 \pm 23.8$ & $27.9 \pm 20.2$ & $23.9 \pm 23.2$ & $13.2 \pm 14.9$ \\
\hline VAS patient pain (mm) & $67.0 \pm 18.0$ & $60.4 \pm 21.5$ & $44.6 \pm 27.1$ & $36.0 \pm 26.7$ & $30.6 \pm 24.8$ & $23.6 \pm 26.3$ \\
\hline
\end{tabular}

\footnotetext{
Data shown as mean $\pm S D$.
}

While there was no difference between patients pretreated with one or two TNF antagonists, patients with three TNF antagonists in the history showed a lesser response which, due to low numbers, was not statistically significant (data not shown)

CRP, C reactive protein; DMARD-IR, inadequate response to disease-modifying antirheumatic drugs; ESR, erythrocyte sedimentation rate; HAQ-DI, Health Assessment QuestionnaireDisease Index; SJC, swollen joint count; TJC, tender joint count; TNF, tumour necrosis factor; TNF antagonist-IR, inadequate response to TNF antagonists; VAS, visual analogue scale. 
Table 2 EULAR and ACR responses in RF-positive and RF-negative patients at week 24

\begin{tabular}{lll}
\hline Efficacy measure & $\begin{array}{l}\text { RF-positive } \\
(\mathbf{n = 1 8 4 )}\end{array}$ & $\begin{array}{l}\text { RF-negative } \\
(\mathbf{n = 8 1 )}\end{array}$ \\
\hline Mean \pm SD DAS28 at baseline & $6.1 \pm 1.0$ & $5.7 \pm 1.1$ \\
Remission (DAS28 <2.6) & $46.2 \%(85)$ & $50.6 \%(41)$ \\
LDAS (DAS28 $\leq 3.2)$ & $57.1 \%(105)$ & $59.3 \%(48)$ \\
Clinically significant DAS28 reduction $\geq 1.2$ & $79.3 \%(146)$ & $70.4 \%(57)$ \\
EULAR good response & $56.0 \%(103)$ & $58.0 \%(47)$ \\
ACR50 & $50.5 \%(93)$ & $50.6 \%(41)$ \\
ACR70 & $33.7 \%(62)$ & $34.6 \%(28)$ \\
\hline
\end{tabular}

Data shown as percentage (n) unless otherwise stated.

ACR, American College of Rheumatology; DAS, Disease Activity Score; EULAR, European League Against Rheumatism; LDAS, Low Disease Activity Score; RF, rheumatoid factor.

were the individual changes clinically significant at the last assessment. Raised aminotransferases were reported in $7.3 \%$ of patients (5.6\% drug-related) and low neutrophil counts in $2.4 \%$ (all drug-related).

\section{DISCUSSION}

TAMARA was the first phase IIIb study preceding the approval of tocilizumab in January 2009 to be performed in a setting close to real-life medical care and confirmed the rapid and sustained improvement in signs and symptoms of RA with tocilizumab as well as a manageable safety profile.

The TAMARA study was conducted in 286 patients with active RA who had received $1-4$ previous DMARDs; in $41.6 \%$ treatment had failed with a TNF antagonist. Concomitant DMARD therapy was continued, which was most often methotrexate $(72.0 \%)$ but $19.6 \%$ of the patients were treated with leflunomide, reflecting the 'real-life' scenario. The high number of protocol deviations also reflects a 'real-life' scenario, but only a few patients had to be withdrawn from the study. Protocol violations did not influence study outcome parameters as the results of the ITT and PP populations were virtually identical.

The TAMARA study confirmed the good efficacy observed in different patient populations in previous studies (see table 4 in the online supplement). ${ }^{11-13} 1516$ Treatment showed a very early onset of action as the mean DAS28 decreased by $40 \pm 20 \%$ after the first tocilizumab infusion. At week $24,57 \%$ of the patients had achieved the primary end point of LDAS and $47.6 \%$ even achieved DAS remission. Remarkably, $79.2 \%$ of the patients in our study had high RA activity with a DAS28 $>5.1$ at baseline. Of important clinical relevance is the fact that tocilizumab treatment was particularly beneficial in these patients, $42 \%$ of whom achieved DAS28 remission by week 24 . Furthermore, the quality of life as reflected by the HAQ-DI, SF-36 and fatigue score also improved. In all, $75 \%$ of the patients reported an overall satisfaction with the treatment.

Because of the strong effect of tocilizumab on the acute phase reactants (APR), it is a matter of debate whether the high remission rates resulting from tocilizumab are mainly driven by the reduction in APR. This can be rebutted by the strong decrease in the number of swollen and tender joints as well as the high percentage of patients (26\%) with no TJC and no SJC (figure 1).
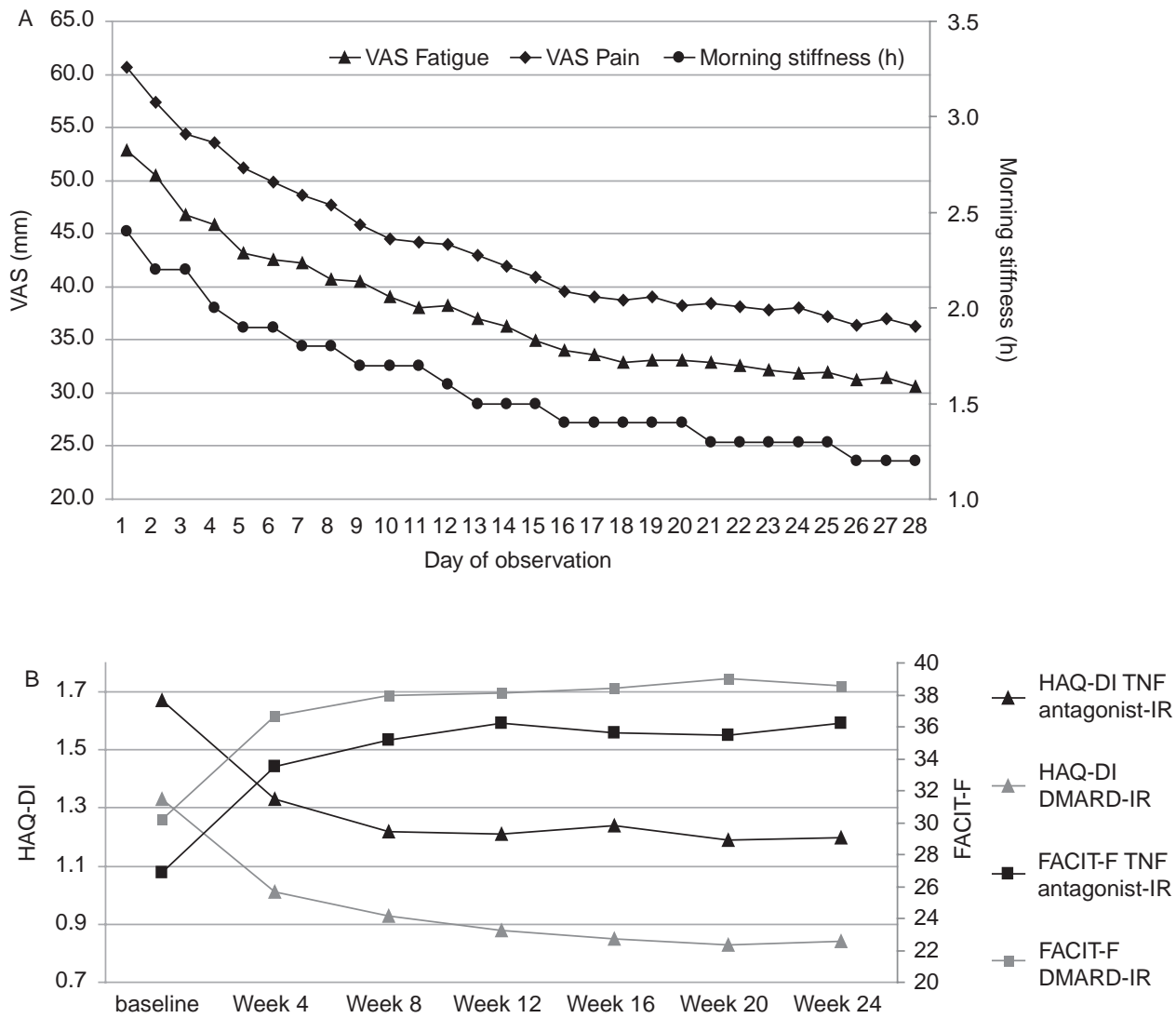

Figure 2 (A) Initial course of VAS fatigue, VAS pain and morning stiffness (patient self-reported) from day 1 to day 28 after only one infusion (LOCF) (values for day 1 were documented after the first infusion). (B) Course of fatigue (FACIT-Fatigue) and HAO-DI in TNF antagonist-IR and DMARD-IR patients. In the HAQ-DI, which had the lowest percentage change, the mean absolute change of 0.48 score points can be considered a clinically relevant improvement. DMARD-IR, inadequate response to disease-modifying antirheumatic drugs; HAO-DI, Health Assessment QuestionnaireDisease Index; LOCF, last observation carried forward; TNF antagonist-IR, inadequate response to TNF antagonists; VAS, visual analogue scale. 
Subgroup analyses revealed a comparable clinical response in RF-positive and RF-negative patients that has not been reported previously. Patients with concomitant leflunomide treatment did not differ from the whole cohort with regard to efficacy parameters and safety, indicating that leflunomide may represent a suitable concomitant DMARD.

Not unexpectedly, patients who had failed with previous DMARDs showed a better clinical response than patients who had shown an inadequate response to previous TNF antagonists, possibly because of their longer disease duration, the earlier use of tocilizumab or because they may have had a less refractory form of RA.

The treatment with tocilizumab was well tolerated. The incidence rates of all AEs (83.9\%) and of drug-related AEs (66.1\%) as well as the incidence of serious infections were as expected from the published reports. Most of the AEs were mild or moderate and resolved by the end of the study. The only unexpected serious AE in this study was a relapse of a pre-existing but previously undiagnosed case of multiple sclerosis (MS) which was rated as drugrelated. This patient had a history of several neurological disorders (including unspecified foci on the MRI), but the diagnosis of MS was not established before the study. Reassessment of the medical charts, however, confirmed the pre-existence of a relapsing-remitting MS and the serious AE was changed to 'MS-relapse'. Thus, a causal relationship with tocilizumab could not be excluded.

This study was performed in a setting close to real-life medical care; however the non-controlled design and the short observation period of 24 weeks provide only limited data with respect to long-term efficacy and potential safety issues. Nevertheless, studies close to real-life medical care in usual clinical practice in a heterogeneous patient population have a high degree of relevance as results of controlled studies do not always match with daily medical care. Only $21-33 \%$ of patients in the German RABBIT register treated with biological agents would have been eligible for the major trials with TNF $\alpha$ antagonists. In the clinical setting, the indications for treatment with biological agents were not identical to the inclusion criteria for trials, and there was a smaller relative improvement achieved in the patients with RA who would not have fulfilled the inclusion criteria of the trials. ${ }^{18}$ Interestingly, the heterogeneous patient population from the TAMARA study showed even better efficacy results than patients from previously published controlled studies (see table 4 in the online supplement). We therefore believe that these data confirm that tocilizumab represents an important treatment option for patients with RA in a real-life setting.

\section{CONCLUSIONS}

This study demonstrated the efficacy of tocilizumab in combination with a traditional DMARD in a heterogeneous patient population with moderate to severe active RA. The good efficacy of tocilizumab was accompanied by a manageable safety profile, which was in accordance with the safety data obtained during the clinical development programme of tocilizumab.

Acknowledgements The authors would like to thank all participating centres and patients as well as Dr Uta Duesberg for supporting the medical writing.

Funding The study was funded by Roche Pharma AG, Grenzach-Wyhlen, Germany and Chugai Pharma Marketing, Frankfurt, Germany.
Competing interests GRB, EF and AR-R are members of advisory boards and received grant support as well as honoraria as speakers from Roche and Chugai. Cl-K is an advisor and received honoraria as a speaker from Roche and Chugai.

Ethics approval This study was conducted with the approval of the Berlin State ethics committee (Berliner Landesethikkommission), EudraCT number 2008000105-11, Investigation Code ML21469.

Provenance and peer review Not commissioned; externally peer reviewed.

\section{REFERENCES}

1. Abbott JD, Moreland LW. Rheumatoid arthritis: developing pharmacological therapies. Expert Opin Investig Drugs 2004;13:1007-18.

2. Hughes LB, Moreland LW. New therapeutic approaches to the management of rheumatoid arthritis. BioDrugs 2001;15:379-93.

3. Moreland LW. Biologic therapies on the horizon for rheumatoid arthritis. J Clin Rheumatol 2004;10(3 Suppl):S32-9.

4. Weaver AL. The impact of new biologicals in the treatment of rheumatoid arthritis. Rheumatology (Oxford) 2004;43:iii17-23.

5. Cohen SB, Moreland LW, Cush JJ, et al. A multicentre, double blind, randomised, placebo controlled trial of anakinra (Kineret), a recombinant interleukin 1 receptor antagonist, in patients with rheumatoid arthritis treated with background methotrexate. Ann Rheum Dis 2004;63:1062-8.

6. Edwards JC, Szczepanski L, Szechinski J, et al. Efficacy of B-cell-targeted therapy with rituximab in patients with rheumatoid arthritis. N Engl J Med 2004;350:2572-81.

7. Kremer JM, Westhovens $\mathrm{R}$, Leon $\mathrm{M}$, et al. Treatment of rheumatoid arthritis by selective inhibition of T-cell activation with fusion protein CTLA4lg. N Engl J Med 2003;349:1907-15.

8. Bathon JM, Martin RW, Fleischmann RM, et al. A comparison of etanercept and methotrexate in patients with early rheumatoid arthritis. N Engl J Med 2000;343:1586-93.

9. Maini RN, Breedveld FC, Kalden JR, et al. Therapeutic efficacy of multiple intravenous infusions of anti-tumor necrosis factor alpha monoclonal antibody combined with low-dose weekly methotrexate in rheumatoid arthritis. Arthritis Rheum 1998;41:1552-63.

10. Olsen NJ, Stein CM. New drugs for rheumatioid arthritis. N Engl J Med 2007;3501:2167-79.

11. Emery P, Keystone E, Tony HP, et al. IL-6 receptor inhibition with tocilizumab improves treatment outcomes in patients with rheumatoid arthritis refractory to antitumour necrosis factor biologicals: results from a 24-week multicentre randomised placebo-controlled trial. Ann Rheum Dis 2008;67:1516-23.

12. Genovese MC, McKay JD, Nasonov EL, et al. Interleukin-6 receptor inhibition with tocilizumab reduces disease activity in rheumatoid arthritis with inadequate response to disease-modifying antirheumatic drugs: the tocilizumab in combination with traditional disease-modifying antirheumatic drug therapy study. Arthritis Rheum 2008;58:2968-80.

13. Jones G, Sebba A, Gu J, et al. Comparison of tocilizumab monotherapy versus methotrexate monotherapy in patients with moderate to severe rheumatoid arthritis: the AMBITION study. Ann Rheum Dis 2010;69:88-96.

14. Nishimoto N, Miyasaka N, Yamamoto K, et al. Study of active controlled tocilizumab monotherapy for rheumatoid arthritis patients with an inadequate response to methotrexate (SATORI): significant reduction in disease activity and serum vascular endothelial growth factor by IL-6 receptor inhibition therapy. Mod Rheumatol 2009;19:12-9.

15. Smolen JS, Beaulieu A, Rubbert-Roth A, et al. OPTION Investigators. Effect of interleukin-6 receptor inhibition with tocilizumab in patients with rheumatoid arthritis (OPTION study): a double-blind, placebo-controlled, randomised trial. Lancet 2008;371:987-97.

16. Fleischmann RM, Burgos-Vargas R, Ambs P. LITHE: Tocilizumab inhibits radiographic progression and improves physical function in rheumatoid arthritis (RA) patients (Pts) at 2 Yrs with increasing clinical efficacy over time. Abstract 637. Oral presentation at American College of Rheumatology, 18 October 2009.

17. Nishimoto N, Hashimoto J, Miyasaka N, et al. Study of active controlled monotherapy used for rheumatoid arthritis, an IL-6 inhibitor (SAMURAI): evidence of clinical and radiographic benefit from an $x$ ray reader-blinded randomised controlled trial of tocilizumab. Ann Rheum Dis 2007;66:1162-7.

18. Zink A, Strangfeld A, Schneider M, et al. Effectiveness of tumor necrosis factor inhibitors in rheumatoid arthritis in an observational cohort study: comparison of patients according to their eligibility for major randomized clinical trials. Arthritis Rheum 2006;54:3399-407. 